\title{
Identification and investigation of ORFans in the viral world Yanbin Yin ${ }^{1}$ and Daniel Fischer*1,2
}

Address: ${ }^{1}$ Computer Science and Engineering Dept, 201 Bell Hall, University at Buffalo, Buffalo, NY 14260-2000, USA and ${ }^{2}$ Bioinformatics/Dept. of Computer Science, Ben Gurion University, Beer-Sheva 84015, Israel

Email: Yanbin Yin - yyin2@cse.buffalo.edu; Daniel Fischer* -df33@cse.buffalo.edu

* Corresponding author

Published: 19 January 2008

BMC Genomics 2008, 9:24 doi:10.1 186/|47|-2/64-9-24
Received: 9 March 2007

Accepted: 19 January 2008

This article is available from: http://www.biomedcentral.com/I47I-2I64/9/24

(C) 2008 Yin and Fischer; licensee BioMed Central Ltd.

This is an Open Access article distributed under the terms of the Creative Commons Attribution License (http://creativecommons.org/licenses/by/2.0), which permits unrestricted use, distribution, and reproduction in any medium, provided the original work is properly cited.

\begin{abstract}
Background: Genome-wide studies have already shed light into the evolution and enormous diversity of the viral world. Nevertheless, one of the unresolved mysteries in comparative genomics today is the abundance of ORFans - ORFs with no detectable sequence similarity to any other ORF in the databases. Recently, studies attempting to understand the origin and functions of bacterial ORFans have been reported. Here we present a first genome-wide identification and analysis of ORFans in the viral world, with focus on bacteriophages.

Results: Almost one-third of all ORFs in I,456 complete virus genomes correspond to ORFans, a figure significantly larger than that observed in prokaryotes. Like prokaryotic ORFans, viral ORFans are shorter and have a lower GC content than non-ORFans. Nevertheless, a statistically significant lower GC content is found only on a minority of viruses. By focusing on phages, we find that $38.4 \%$ of phage ORFs have no homologs in other phages, and $30.1 \%$ have no homologs neither in the viral nor in the prokaryotic world. Phages with different host ranges have different percentages of ORFans, reflecting different sampling status and suggesting various diversities. Similarity searches of the phage ORFeome (ORFans and non-ORFans) against prokaryotic genomes shows that almost half of the phage ORFs have prokaryotic homologs, suggesting the major role that horizontal transfer plays in bacterial evolution. Surprisingly, the percentage of phage ORFans with prokaryotic homologs is only $18.7 \%$. This suggests that phage ORFans play a lesser role in horizontal transfer to prokaryotes, but may be among the major players contributing to the vast phage diversity.
\end{abstract}

Conclusion: Although the current sampling of viral genomes is extremely low, ORFans and nearORFans are likely to continue to grow in number as more genomes are sequenced. The abundance of phage ORFans may be partially due to the expected vast viral diversity, and may be instrumental in understanding viral evolution. The functions, origins and fates of the majority of viral ORFans remain a mystery. Further computational and experimental studies are likely to shed light on the mechanisms that have given rise to so many bacterial and viral ORFans. 


\section{Background}

Genome-wide studies of microbial ORFans have been carried out for about ten years now $[1,2]$. One of the major surprises in these studies is the large percentage of ORFs (Open Reading Frames) having few or no homologs in the databases $[3,4]$. These are referred to as ORFans [1]. With hundreds of complete prokaryote genome sequences, it has become evident that the presence of many ORFan genes is a natural phenomenon [3-7], that will continue to be observed for years. Recent studies have suggested that most ORFans are likely to correspond to real, expressed and functional proteins [8-13]. Despite studies focusing on particular bacterial lineages [14], the origin and functions of ORFans remain a mystery $[6,15,16]$ : If proteins in different organisms have descended from common ancestral proteins by duplication and adaptive variation, why is it that so many today show no similarity to each other $[4,15]$ ? Why is it that we do not find today any of the necessary "intermediate sequences" that must have given rise to these ORFans? Do most ORFans correspond to rapidly diverging proteins $[17,18]$ ? If so, do they mainly correspond to nonessential proteins or to the species determinants?

Regardless of their origin, ORFans may be of two types [4]. Some ORFans may correspond to proteins with unique functions not currently observed in other families. Alternatively, ORFans may correspond to rapidly evolving and highly divergent members of known protein families, but with functions similar to proteins already known. Notice that our ORFan definition does not depend on database annotations beyond the mere ORF identification or on assumptions about the origin of ORFans. We use a merely operational definition: an ORFan is simply an ORF with no detectable sequence similarity to other ORFs in the database considered. This definition allows us to objectively identify and quantify ORFans, a required first step towards attempting to understand their functions and origins.

Recently, there has been a renewed interest in viral genomics [19], in part because of the realization of the major role that viruses, and in particular phages, have played in evolution. So far, the complete genome sequences of more than a thousand viruses, including hundreds of phages, have been deposited in the public database. Recent studies of phage genomes have revealed that horizontal transfer (HT) has played a major role in viral genome evolution $[20,21]$. Phages exchange genes with other phages mostly when they are inside the same host cell and with prophages residing in the host genome $[22,23]$. Phages can also exchange genes with their hosts, by integrating them as prophages or by exchanging individual genes with their hosts via recombination [24-26]. In particular, recent sequencing and comparative analyses of cyanophages and cyanobacteria has revealed some cases of HTs from hosts to phages [27-29].

Here we extend our ORFan studies to the viral world by addressing the following questions: 1 ) what is the percentage of ORFans in viruses and phages, how does it compare to the percentage of ORFans in bacteria, and how is this percentage related to the current phage sampling and to phage diversity? 2) Do viral ORFans show particular characteristics regarding length and GC content, as bacterial ORFans do? 3) How scarce is the current viral sampling, and are current observations likely to hold after many more genomes are sequenced? 4) What role do phage ORFans play in horizontal transfer from and to bacterial hosts?

\section{Results and discussion}

The viral sequence data used in this work was obtained by downloading all 1,456 virus genomes available at Refseq [30] on September 2005. These genomes encode a total of 43,566 ORFs. We refer to this set of ORFs as our "AllVirus-DB". Out of the 1,456 virus genomes, 280 are phages, encoding a total of 18,368 ORFs. We refer to this set of phage ORFs as our "Phage-DB".

\section{ORFans collection}

A viral ORF is defined to be a viral ORFan if a BLASTP search against our All-Virus-DB finds no significant hits outside its residing genome (see Material and Methods). $13,078(30.0 \%)$ viral ORFs were thus identified as viral ORFans, a figure similar to that previously reported [31]. Notice that the percentage of viral ORFans is much larger than that of bacterial ORFans $(9.1 \%$ in [6]). This confirms previous observations suggesting that the diversity among viruses is expected to be significantly larger than that among bacteria $[31,32]$.

It is important to point out that our identification of ORFans is highly dependant on the quality of gene identification programs used to generate the list of ORFs in the database, especially the shorter ones. ORFs may be underpredicted (false negatives in gene prediction, and thus should be present in the database), or over-predicted (false positives in gene prediction, and thus should not be present in the database). It is beyond the scope of this paper to attempt to improve the ORF identification programs or to estimate the ratio of under- versus over- predicted ORFs in vira. Nevertheless, to partially overcome the problem of over-prediction of short ORFs that may not correspond to real genes, we have repeated all computations considering only ORFs longer than 300 bp (see Dataset Controls in Methods below). Previous works have shown that it is more unlikely for a longer ORF to be a false positive $[3,33]$. We believe that using this length threshold, we have significantly reduced the number of 
possible short false positives without removing a significant number of true-positive short ORFs.

\section{Special characteristics of ORFans}

It has been observed that bacterial ORFans have a number of characteristics different than those found in bacterial non-ORFans $[3,14,34]$. One of these is length: bacterial ORFans are shorter than non-ORFans on average (mean value 159 vs. 327 residues; p-value $<2.2 \mathrm{e}-16$ ). To test whether viral ORFans are also shorter than viral nonORFans, we computed the length distribution of viral ORFans and non-ORFans (Figure 1). As is clear from the figure, viral ORFans are also shorter than non-ORFans, with mean length values similar to those of Bacteria (172 vs. 356 residues; $\mathrm{p}$-value $<2.2 \mathrm{e}-16$ ).

Another reported characteristic of bacterial ORFans is that they have significant lower GC content than non-ORFans $[14,34]$. The GC3 content (GC at the third codon positions [34]) of bacterial ORFans is $51.4 \%$ whereas that of bacterial non-ORFans is $54.2 \%$ ( $\mathrm{p}$-value $<2.2 \mathrm{e}-16)$. To test whether this also holds for viruses, we computed the average GC3 content of viral ORFans and compared it to that of viral non-ORFans. The mean GC3 content of viral ORFans is $44.7 \%$, also significantly lower than that of viral non-ORFans (45.9\%; p-value $=7.0 \mathrm{e}-13)$. Nevertheless, notice that the difference in GC3 content between

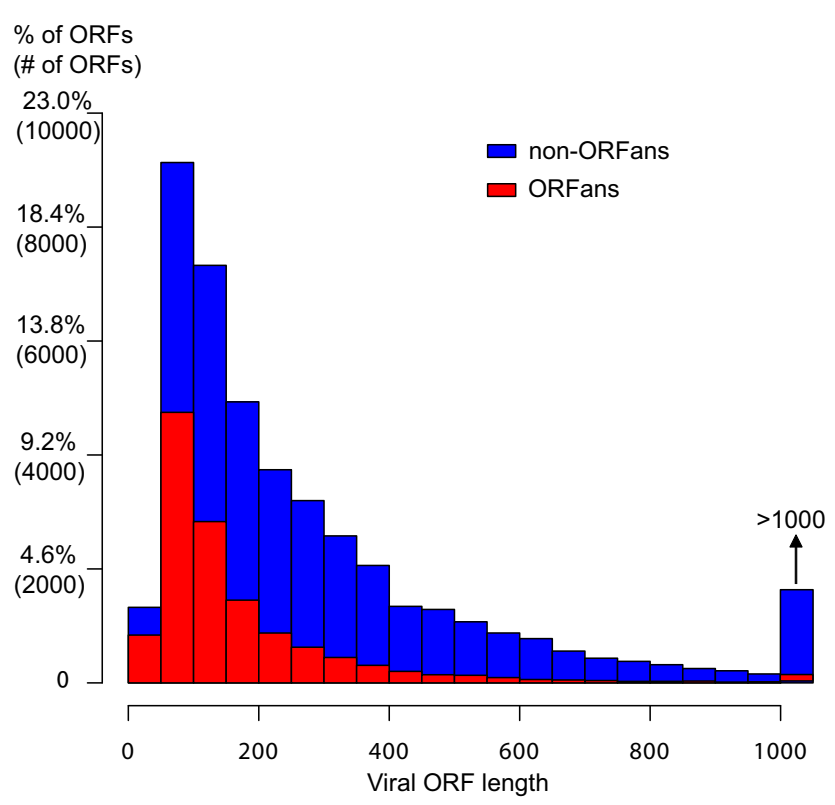

\section{Figure I}

Viral ORFans are shorter than non-ORFans on average. The mean ORF length of ORFans is 172, whereas that of nonORFans is 356 . The histogram shows the length distribution of all viral ORFs. The lower bars correspond to viral ORFans.
ORFans and non-ORFans is smaller and statistically less significant in viruses than it is in bacteria.

However, it is important to point out that using averages can be dangerous, as averages do not take into account the properties of individual genomes. While the observation that ORFans are shorter on average than non-ORFans holds for the vast majority of individual genomes, the observation that ORFans have lower GC content does not. The percentages of individual genomes that show that ORFans are statistically significantly shorter than nonORFans are $99.6 \%$ and $100 \%$, among bacterial and viral genomes, respectively (Figure 2A-B). But the percentages of individual genomes that show that ORFans have statistically significant lower GC content than non-ORFans are only $70.2 \%$ and $26.8 \%(!)$ among the bacterial and viral genomes, respectively (Figure 2C-D).

\section{Focusing on phages: higher percentage of ORFans in phages}

The 1,456 genomes in our All-Virus-DB comprise a very diverse set of organisms as it includes a variety of distantly related viral species with very diverse lifestyles: the 3 most abundant virus classes are ssRNA positive-strand viruses with no DNA stage (450 genomes), dsDNA viruses with no RNA stage (425 genomes), and ssDNA viruses (244 genomes). In addition, the viruses in our All-virus-DB show a great variability in genome size. Figure 3 shows the distribution of genome size (measured as the number of ORFs per viral genome) in our All-virus-DB. The average number of ORFs per genome is 30 . Most of the viruses have very small genomes: $73.3 \%$ of the viruses have genomes with fewer than 20 ORFs ( 85 genomes have only one ORF), while only $7.4 \%$ of the viruses have more than 100 ORFs (the 3 largest genomes are: Acanthamoeba polyphaga mimivirus, Paramecium bursaria Chlorella virus and Shrimp white spot syndrome virus with 911, 690 and 531 ORFs, respectively).

The red bars in Figure 3 correspond to those viruses identified as phages (280 in total). Compared with the other viruses, phages have less extreme distribution of number of ORFs per genome: 163 out of the 280 ( 60\%) phages have between 40 and 100 ORFs in their genome. In addition, phages are mainly restricted to one taxonomical class: $75 \%$ of phages (211) correspond to dsDNA viruses with no RNA stage. In what follows, we study these 280 phages in more detail. We refer to the set of 280 phages as our "Phage-DB", which contains a total of 18,368 ORFs. Notice that phages correspond to less than 20\% (280/ 1456) of all viral genomes, while the total number of phage ORFs correspond to more than $42 \%(18,368 /$ $43,566)$ of all viral ORFs. 

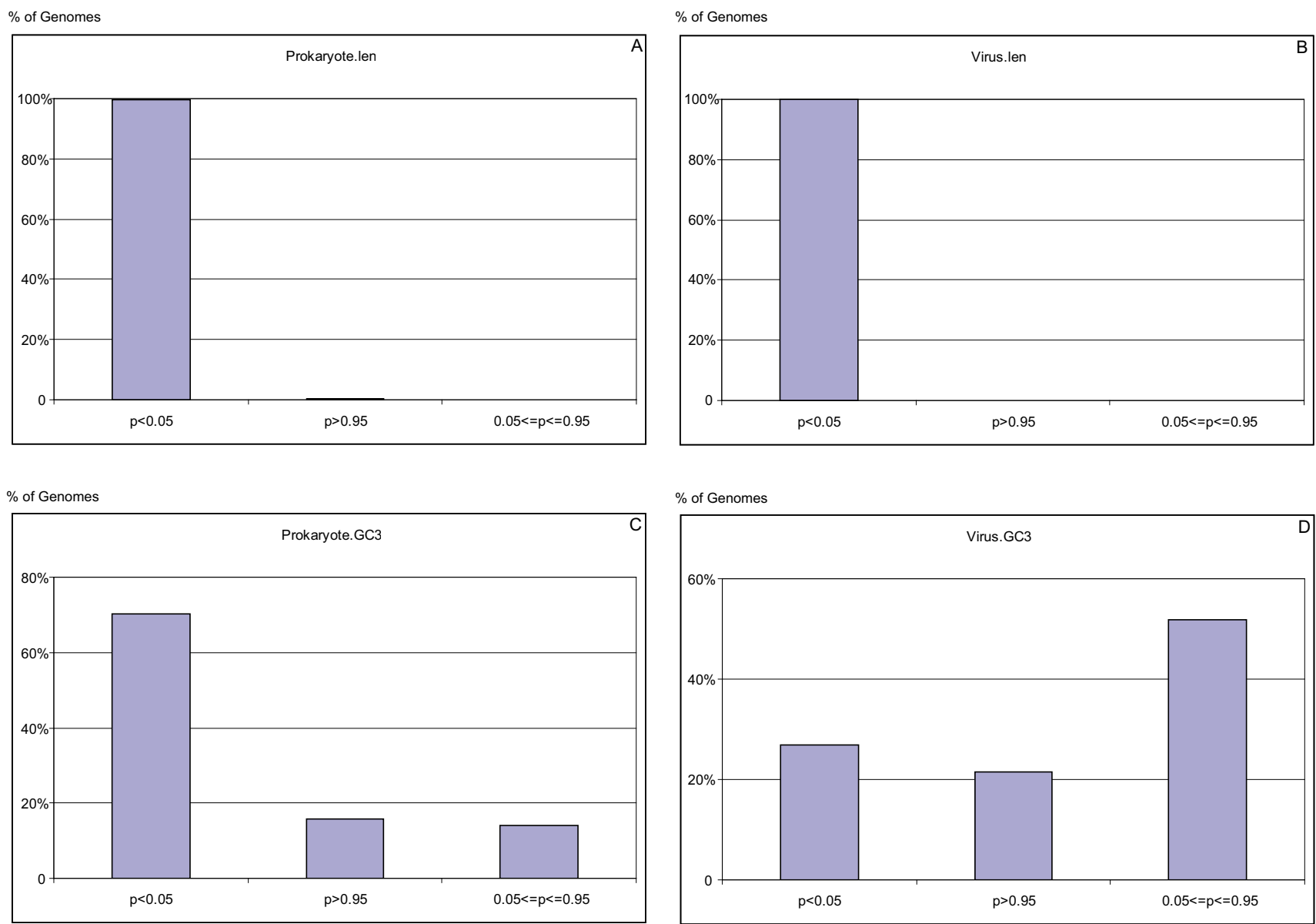

\section{Figure 2}

Length and GC3 content of ORFans and non-ORFans. While ORFans are statistically significantly shorter than non-ORFans in the majority of genomes, they have statistically significantly lower GC3 content than non-ORFans in a smaller fraction of the genomes. We applied the Wilcoxon nonparametric test to individual genomes. The null hypotheses were that ORFans are not shorter than non-ORFans, and that they do not have lower GC3 content than non-ORFans. For each genome, we compared the protein length and GC3 content distributions of its ORFans and non-ORFans. A p-value $<0.05$ rejects the null hypothesis, and significantly supports the alternative hypothesis. A p-value $>0.95$ supports the null hypothesis and a $p$-value between the two values means no significant difference. Only genomes with at least 30 ORFans and 30 non-ORFans were considered. The upper panels show the results of the length test on prokaryotes (left) and viruses (right). The lower panels show the results of the GC3 content test on prokaryotes (left) and viruses (right). Clearly, while the lower GC3 content of ORFans is statistically significant for about $70 \%$ of the bacterial genomes, it is statistically significant only for $27 \%$ of the viral genomes.

Out of 18,368 phage ORFs, 7,047 (38.4\%) correspond to phage ORFans (no homologs in other phages), significantly larger than the percentage of ORFans among all viruses $(30.0 \%)$. This suggests that the diversity among phages is larger than that among all viruses. Interestingly, all but $282(4.0 \%)$ out of the 7,047 phage ORFans correspond to viral ORFans too (no homologs in All-VirusDB).

\section{Growing number of ORFans}

Figure 4 shows the growth in the number of ORFans and the decrease in the percentage of ORFans as a function of the number of available phage genomes. The figure suggests that although the percentage of ORFans is gradually decreasing, it is not likely to drop significantly even after hundreds of more phage genomes are sequenced. It also suggests that the actual number of ORFans will continue to grow as more genomes are sequenced. These trends in phages are consistent with those observed in prokaryotes $[4,5]$.

To estimate the dependence of the number of ORFans to our database of fully sequenced viruses, we have searched for homologs of our identified viral ORFans within the 


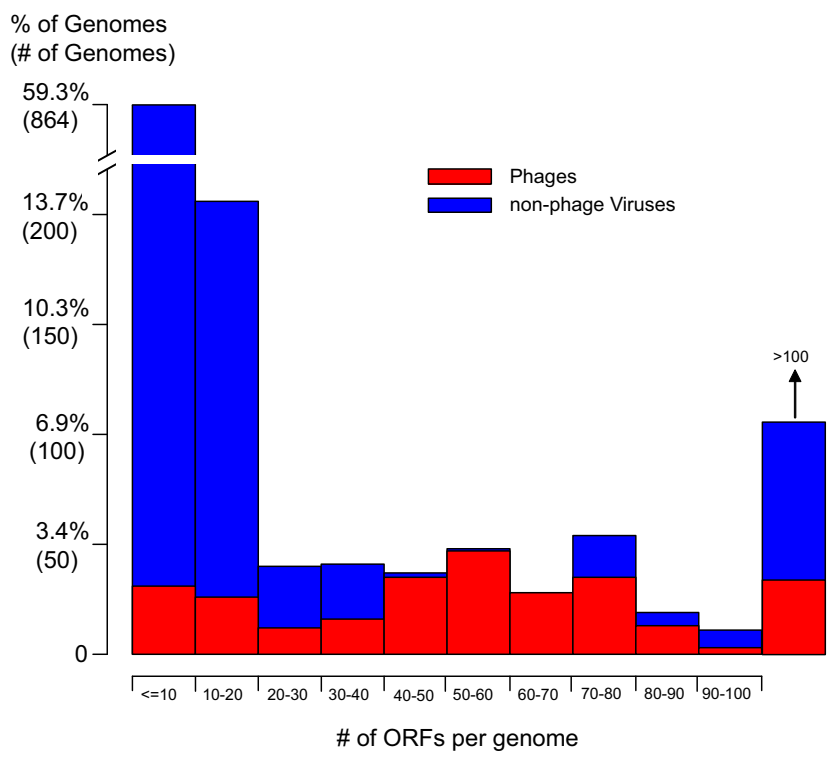

Figure 3

Distribution of the number of ORFs per genome in I,456 viruses. Phages have a less extreme distribution of number of ORFs per genome than that of the non-phage viruses.

recent (as of May 22, 2007) nr [35] and env_nr [36] (which includes the 6 million recently published predicted marine metagenomic proteins [37]) databases. We found that out of our 13,078 identified viral ORFans, 4878 have homologs in these databases. This demonstrates that the abundance of current ORFans is partially due to the number of fully sequenced organisms, and that a fraction of current ORFans will become non-ORFans as

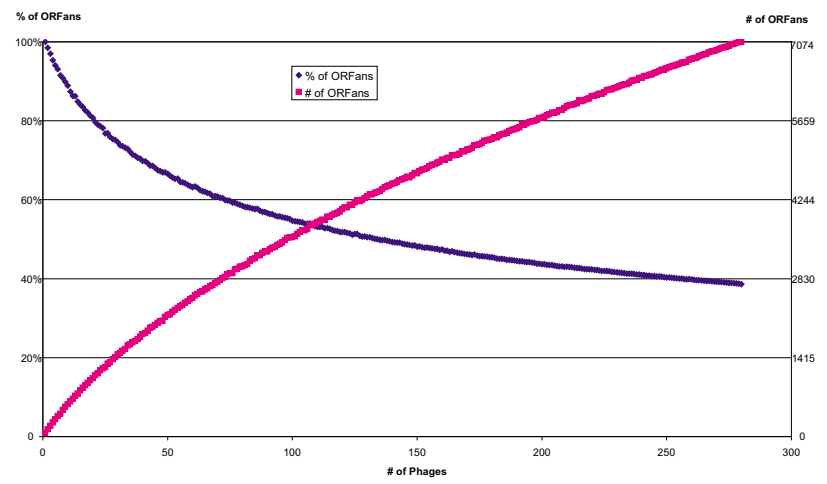

Figure 4

The number of phage ORFans is growing. The plot was computed by averaging the results of 1000 random selections of subsets of the 280 phage genomes. The average number of ORFans in each subset size is shown in red, and the percentage of ORFans (computed as the number of ORFans divided by the total number of ORFs) is shown in blue. more complete genomes are sequenced. However, as we have previously demonstrated [3], the number of new ORFans in newly sequenced genomes is greater than the number of older ORFans that become non-ORFans. Indeed, it is estimated that $>91 \%$ marine viral genes are novel [38]. Consequently, as more complete genomes are sequenced, the total number of ORFans will continue to grow, and our figure of $30.0 \%$ viral ORFans is not likely to vary dramatically in the coming years [4].

\section{ORFans in different phage groups}

It has been estimated that each microbial species is a host for at least 10 phage species, suggesting the phage diversity is at least 10 times higher than microbial diversity, comprising about $10^{8}$ species [39]. Nevertheless, there are only 280 phage genomes in our Phage-DB, suggesting that at least to some extent, the very high percentage of phage ORFans is a result of low phage sampling. To further explore this possibility, we studied the ORFans percentages in different phage groups.

Because phages with phylogenetically close hosts are often similar to each other [22,40-44], we classified the 280 phages according to their host ranges. We identified the hosts attacked by these phages, by automatically parsing the corresponding Genbank format files, or manually checking the related literature and searching against NCBI taxonomy database (Additional file 1). In Table 1, we list the taxonomical groups of the hosts identified, showing the number of sequenced prokaryotes, the number of sequenced phages, the sampling ratio and the percentage of phage ORFans. The table lists the 12 major lower level groups (those with less than 5 phages are grouped as "Others"), and the three higher level groups: Firmicutes, Gamma-Proteobacteria, and "non-Firm-Gamma". The average phage sampling ratio in each group is computed as the number of prokaryotes divided by the number of phages. This ratio reflects the current sampling status for each phage group. A sampling ratio of 10 would suggest that microbes are infected by 10 phages on average. However, the overall average ratio for all phages is only slightly higher than 1.0 (280 phages/277 prokaryotes), clearly showing that the current phage sampling is very low; the highest ratio is 3.3 for the Sulfolobales group, and the lowest (excluding "Others") is 0.38 for the Cyanobacteria group. The table also shows that the current sampling is biased towards Firmicutes and Gamma-Proteobacteria, the two most prevalently studied bacteria phyla [6]. In addition, within each phage group, the sampling is also biased, often toward some intensively studied bacteria. For example, 43 out of 62 Enterobacteria phages infect Escherichia coli; 35 out of 48 Bacillales phages infect Staphylococcus aureus and 12 out of 18 Pseudomonadales phages infect Pseudomonas aeruginosa (Additional file 1). 
Table I: Prokaryotes/Phage groups classified by phage host ranges and the percentage of ORFans within each group

\begin{tabular}{|c|c|c|c|c|}
\hline Prokaryotic/Phage group & \# of prokaryotes & \# of phages & Sampling ratio & $\%$ of Phage ORFans \\
\hline Bacteria_Proteobacteria_Gammaproteobacteria_Enterobacteriales & 24 & 62 & 2.58 & $33.0 \%$ \\
\hline Bacteria_Firmicutes_Bacilli_Bacillales & 27 & 48 & 1.78 & $26.1 \%$ \\
\hline Bacteria_Firmicutes_Bacilli_Lactobacillales & 20 & 44 & 2.20 & $31.9 \%$ \\
\hline Bacteria_Actinobacteria_Actinobacteria_Actinobacteridae & 19 & 20 & 1.05 & $56.6 \%$ \\
\hline Bacteria_Proteobacteria_Gammaproteobacteria_Vibrionales & 6 & 18 & 3.00 & $77.6 \%$ \\
\hline Bacteria_Proteobacteria_Gammaproteobacteria_Pseudomonadales & 9 & 18 & 2.00 & $88.7 \%$ \\
\hline Bacteria_Proteobacteria_Betaproteobacteria_Burkholderiales & 9 & 15 & 1.67 & $41.7 \%$ \\
\hline Archaea_Crenarchaeota_Thermoprotei_Sulfolobales & 3 & 10 & 3.33 & $53.2 \%$ \\
\hline Bacteria_Firmicutes_Mollicutes & 14 & 9 & 0.64 & $64.8 \%$ \\
\hline Bacteria_Cyanobacteria & 13 & 5 & 0.38 & $57.0 \%$ \\
\hline Bacteria_Chlamydiae & 10 & 5 & 0.50 & $6.8 \%$ \\
\hline Others & 123 & 26 & 0.21 & \\
\hline All Gamma & 63 & 109 & 1.73 & $40.2 \%$ \\
\hline All Firmicutes & 66 & 102 & 1.55 & $27.1 \%$ \\
\hline All non-Firm-Gamma & 148 & 69 & 0.47 & $53.7 \%$ \\
\hline
\end{tabular}

Table 1 also shows that the percentage of ORFans varies significantly among the groups (last column). Firmicutes have the fewest proportion of ORFans $(27.1 \%)$ and "nonFirm-Gamma" have the largest (53.7\%), reflecting the fact that the lowest sampled group has the highest percentage of ORFans. We would expect to observe a large negative correlation between the phage sampling ratios and the percentage of ORFans, if the following three conditions held: 1) the high percentage of ORFans is mainly influenced by the sampling; 2) different phage groups have similar diversities and 3 ) the phages that infect prokaryotes are evenly distributed. However, we do not observe any correlation between the phage sampling ratios and the percentage of ORFans (Spearman's rank correlation rho $=-0.17$, p-value $=0.61$. This may be mainly due to the biased phage sampling and the varying diversities among the groups. For example, the Pseudomonadales phages contain the highest percentage of ORFans $(88.7 \%)$, despite having a relatively large sampling ratio (18 phages for 9 bacteria). This suggests a relatively high phage diversity in this group [42]. In contrast, the Chlamydiae phages contain the lowest percentage of ORFans (6.8\%), despite having a relatively low sampling ratio (5 phages for 10 bacteria). Interestingly, these 5 phages are all ssDNA viruses, whose diversity was not thought to be as high as that of dsDNA phages [43].

In summary, the above analysis shows that the current phage sampling is biased and scarce, corroborating previous observations $[6,39]$. Thus, any conclusions derived from the current data should be taken as preliminary observations only. Nevertheless, we believe that the data today already allows us to begin genome-wide studies, and some major trends already observed may be highly informative and may hold, at least qualitatively, when many more phages are sequenced.

\section{ORF conservation in the viral and prokaryotic world}

We investigate the degree of conservation of phage ORFs, measured as the number of detectable phage homologs per ORF. For each ORF o, we compute its $\mathrm{H}$ value, defined as the number of phage genomes that contain homologs to o [6]. Notice that ORFs with $\mathrm{H}=1$ correspond to ORFans, and low values of $\mathrm{H}$ correspond to narrowly distributed ORFs and near ORFans. Figure 5 (left panel) shows the histogram of $\mathrm{H}$-value percentages $(\mathrm{H}$ value divided by the total number of phages: 280) for all phage ORFs.

The figure shows that the phage ORFeome is highly diverse: in addition to the large percentage of ORFans (38.4\%; $\mathrm{H}=1)$, about a third $(32.4 \%, 5,959$ ORFs) of all phage ORFs have homologs in less than 5 phages $(2<=$ $\mathrm{H}<=5$ ), and no phage ORF is conserved in more than $30 \%(\mathrm{H}>84)$ of all the phages. As a comparison, the right panel shows the corresponding $\mathrm{H}$-value percentage plot for prokaryotic ORFs, computed using a database of 277 prokaryotic genomes containing a total of 820,768 ORFs [6]. The figure shows that the prokaryotic ORFeome is less diverse than the phage ORFeome: the percentage of ORFans and near-ORFans are much lower than in phages (9.1\% ORFans $[\mathrm{H}=1]$ and $11.3 \%$ near ORFans $[2<=\mathrm{H}<$ $=5]$ ) and $42.6 \%$ of bacterial ORF are conserved in at least $30 \%$ of the genomes.

\section{HT between viral and prokaryotic worlds}

It has been observed that there is a large phage gene pool residing inside prokaryotes (as prophages or phagederived dispersed genes), suggesting extensive horizontal 

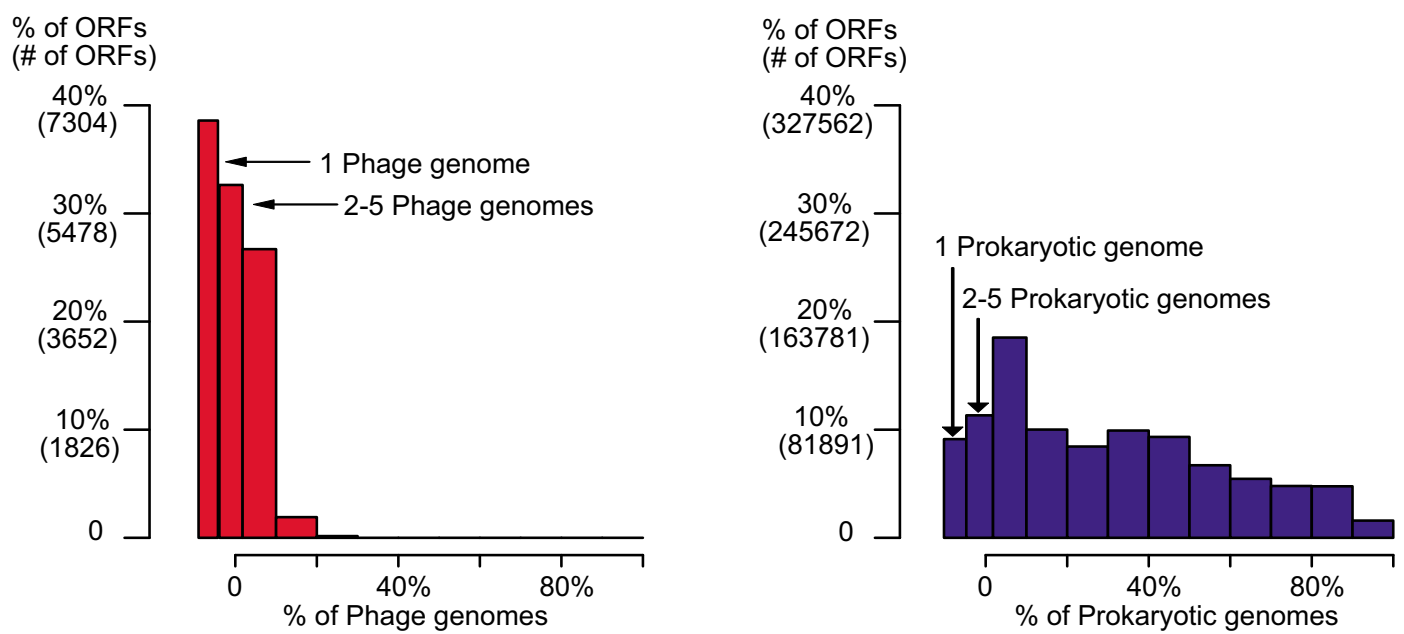

\section{Figure 5}

Histograms of $\mathrm{H}$-value percentages for all phage ORFs (left) and all prokaryotic ORFs (right). The width of bins showing ORFans (I phage/prokaryotic genome) and near-ORFans (2-5 phage/prokaryotic genomes) are expanded. Comparison of the two distributions clearly shows that there is a much lower conservation among phages than in prokaryotes.

transfer (HTs) from phages to the hosts (phage-to-host HTs) $[25,26]$. In addition, HTs of host genes into phages (host-to-phage HTs) have also been observed [29,45]. Therefore, we should expect to find prokaryotic homologs for those phage ORFans and non-ORFans involved in HT. That is, some of the phage ORFans are not absolute ORFans when considering the prokaryotic ORFeome. To attempt to identify and quantify HTs between the two worlds, we carried out BLAST searches for each phage ORF (ORFans and non-ORFans) within the 280 phages in our Phage-DB against our database of 277 complete prokaryotic genomes. Figure 6 depicts our results as a function of the phage ORF H-value computed above.

Considering phage ORFans only, we found prokaryotic homologs for only $18.7 \%$ of the phage ORFans $(1,317$ out of 7,047, blue and green bars in the first column of Figure 6). Interestingly, the corresponding computation in prokaryotes showed that only $1.7 \%$ of the bacterial ORFans have homologs in the viral world [6]. By focusing on the best prokaryotic match, we find that $44.7 \%$ of these ORFans' best homologs (589 out of 1,317, green bars) correspond to prophages within a prokaryotic genome, clearly indicating that they correspond to phage-to-host HTs. In contrast, we found prokaryotic homologs for $63.8 \%$ of the phage non-ORFans $(7,150$ out of the 11,212 phage non-ORFans, blue and green bars in the other columns), and $61.5 \%$ of these non-ORFans' best prokaryotic homologs $(4,397$ out of 7,150$)$ correspond to prophages. This shows that (i) the percentage of phage non-ORFans with prokaryotic homologs is significantly higher than that of the phage ORFans ( $63.8 \%$ vs. $18.7 \%$ ) and that (ii) the more conserved a phage ORF is within the phage world (higher H-value), the more likely it is to have homologs in the prokaryotic world, and the more likely it is that its best homolog resides within a prophage; in particular, all of the phage ORFs $(1,624)$ with $\mathrm{H}>=15$ have homologs in prokaryotes $(80.4 \%$ of which reside in prophages). These findings are consistent with those recently observed in mycobacteriophages [44]. We propose that this may be a consequence of the simple fact that a better conserved phage ORF (with a larger H-value) means that our database contains more phages that can potentially transmit a homolog to prokaryotes. Considering phage ORFans and non-ORFans together, we find that $46.4 \%(8,467)$ phage ORFs have homologs in the prokaryotic world, of which $58.9 \%(4,987)$ have their best homologs within prophages. This suggests that over one fourth of the phage ORFs have been involved in HT from the phage to the bacterial world.

While determining HT from phage to bacteria on the basis of finding prophage homologs is relatively straightforward, other types of HT (host to phage HT or phage to host HT, but not as prophages) are more difficult to identify. While some studies have already identified cases of host to phage HT [27], further studies are required to estimate the overall fraction of phage ORFans and nonORFans that correspond to horizontally transferred ORFs from bacteria. The fact that the percentage of ORFans with bacterial homologs is significantly lower than that of nonORFans suggests that many phage ORFans may corre- 


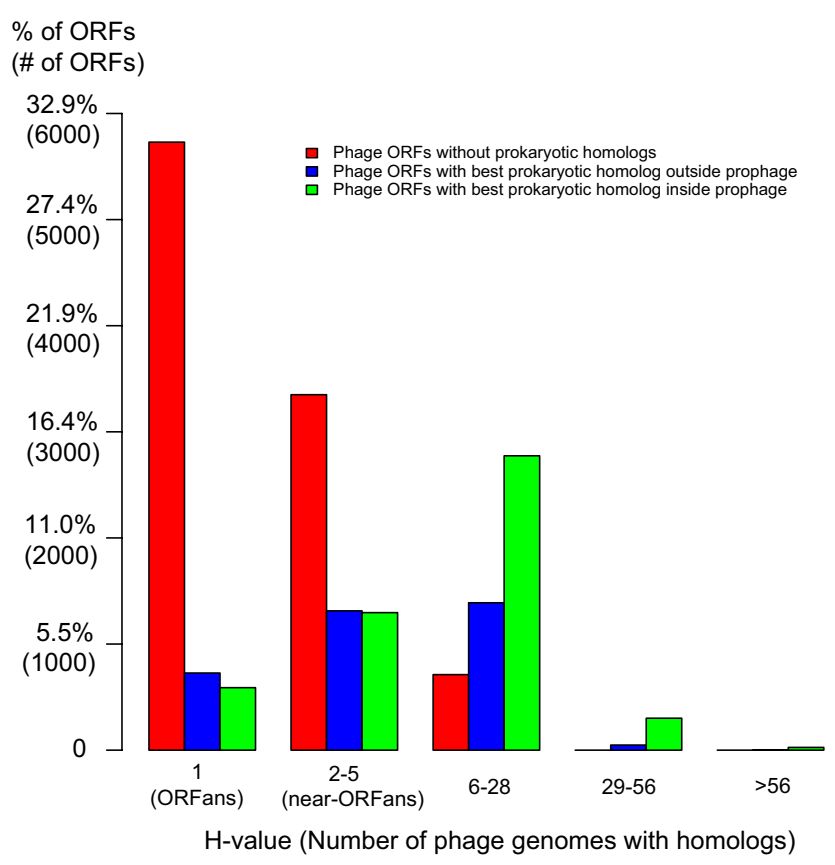

Figure 6

Bar plot of the $\mathrm{H}$-value percentages of phage ORFs. On the basis of the left panel of Fig. 5, three types of phage ORFs are shown. Red bins correspond to phage ORFs with no prokaryotic homologs. The other bins correspond to phage ORFs having homologs in prokaryotes, which include two types: those with their best homolog not in prophages (blue) and those with their best homolog within prophages (green). The less conserved a phage ORF is within phages, the less likely it is to be a prokaryotic homolog. In addition, the more conserved a phage ORFs that has a prokaryotic homolog is, the more likely it is that its best homolog resides as a prophage.

spond to phage specific functions. Thus phage ORFans will likely turn out to be major players in the enormous phage diversity. Further sampling of bacterial and phage genomes will undoubtedly contribute to a better understanding of the evolution and the relationship between these two worlds.

\section{Conclusion}

We have carried out a first systematic analysis of viral ORFans. We have found that the percentage of ORFans in the virus world is much higher than the percentage of ORFans among bacteria. We have found that, like in the bacterial world, viral ORFans are shorter than nonORFans on average, and that this difference is statistically significant in the vast majority of individual genomes. We have also found that, like in the bacterial world, viral ORFans have a lower GC3 content than non-ORFans on average. However, when studying individual genomes we found that the difference is statistically significant only for a small percentage of the individual phage genomes. We found that while a majority of phage non-ORFans have bacterial homologs $(61.5 \%$ of which are likely to be involved in phage to host horizontal transfer), only a small percentage of phage ORFans have bacterial homologs (and only a small fraction of which are involved in phage to host HT). Because the current sampling of phages (and of bacterial genomes in general), is limited and biased towards particular groups, the percentage of ORFans in different phage groups varies significantly. This low sampling may be a factor contributing to the abundance of phage ORFans, but is not likely to be the only one. That is, even after many more genomes are sequenced, we expect to find a significant number of ORFans and near-ORFans, awaiting interpretation. There are also other possibilities to account for the ORFans' origin, like rapid divergence after horizontal transfer (from hosts or from other viruses, from existent genomes or yet extinct genomes) or duplication. Future studies are required to elucidate how much these possibilities account the most for what viruses: e.g. marine phages have been thought to have grasped many genes from their hosts [27]. Unraveling the mystery of the origins and functions of ORFans will likely remain a major challenge.

\section{Methods \\ Protein and genomic data}

Virus proteins and genomic data in Refseq release 13 (Sept. 13, 2005) were downloaded from [46]. 43,566 viral proteins are cross referenced to 1,456 NCBI taxonomical species. Another 72 viral species not encoding proteins in the Refseq release 13 were excluded in our analyses. 277 fully sequenced microbial proteomes and genomes (Nov. 03, 2005) were downloaded from [47], as described in [6].

\section{ORFan identification}

Viral ORFans were identified using a procedure similar to the one we have used in previous work [6]: A viral ORF is defined to be a viral ORFan if a BLASTP search against our All-Virus-DB finds no significant hits outside its residing genome. We define a significant hit if the BLASTP e-value is lower than $1 e-3$ (or for alignment lengths < 80aa, 1e-5).

\section{Prophage identification}

We identified prophages residing in the prokaryotic genomes using our own perl scripts implementing a method similar to that used by Bose and Barber [48].

\section{Dataset controls}

To investigate the possible dependence of our results to the particular data used, and to attempt to quantify the extent of any present bias, we have carried out three control experiments. In each control, we applied the exact same computations as the ones described in the text, and 
verified that the results remain statistically significant. The three control experiments exclude subsets of the data as follows: a) Excluding shorter ORFs $(<=300 \mathrm{bp}) \mathrm{b})$ Excluding smaller genomes ( $<50$ ORFs) and c) Excluding ssDNA and all RNA viruses. In all three cases, our main observations remain statistically significant. A detailed summary of each of these controlled can be found at [49].

\section{Authors' contributions}

YY and DF designed the research. YY conducted the computation, analyzed data, and drafted the manuscript. DF supervised this project and finalized the manuscript. All authors read and approved the final manuscript.

\section{Additional material}

\section{Additional File 1}

280 fully sequenced phages and their hosts. Taxonomy information and corresponding bacterial host for each phage.

Click here for file

[http://www.biomedcentral.com/content/supplementary/1471-

2164-9-24-S1.xls]

\section{Acknowledgements}

Work in the Fischer lab is supported by grants from the NIH and from the EU commission.

\section{References}

I. Fischer D, Eisenberg D: Finding families for genomic ORFans. Bioinformatics 1999, I 5(9):759-762.

2. Dujon B: The yeast genome project: what did we learn? Trends Genet 1996, I 2(7):263-270.

3. Siew N, Fischer D: Analysis of singleton ORFans in fully sequenced microbial genomes. Proteins 2003, 53(2):24I-25I.

4. Siew N, Fischer D: Twenty thousand ORFan microbial protein families for the biologist? Structure (Camb) 2003, I I (I):7-9.

5. Wilson GA, Bertrand N, Patel Y, Hughes JB, Feil EJ, Field D: Orphans as taxonomically restricted and ecologically important genes. Microbiology 2005, I 5 I (Pt 8):2499-250I.

6. Yin Y, Fischer D: On the origin of microbial ORFans: Quantifying the strength of the evidence for viral lateral transfer. BMC Evol Biol 2006, 6(I):63.

7. Leplae R, Lima-Mendez G, Toussaint A: A first global analysis of plasmid encoded proteins in the ACLAME database. FEMS microbiology reviews 2006, 30(6):980-994.

8. Alimi JP, Poirot O, Lopez F, Claverie JM: Reverse transcriptasepolymerase chain reaction validation of 25 "orphan" genes from Escherichia coli K-I2 MG I655. Genome Res 2000, I 0(7):959-966.

9. Monchois V, Abergel C, Sturgis J, Jeudy S, Claverie JM: Escherichia coli ykfE ORFan gene encodes a potent inhibitor of C-type lysozyme. J Biol Chem 200I, 276(2I): I8437-I844I.

10. Shmuely H, Dinitz E, Dahan I, Eichler J, Fischer D, Shaanan B: Poorly conserved ORFs in the genome of the archaea Halobacterium sp. NRC-I correspond to expressed proteins. Bioinformatics 2004, 20(8): I 248-I 253.

II. Zhang R, Joachimiak G, Jiang S, Cipriani A, Collart F, Joachimiak A: Structure of phage protein BCI 872 from Bacillus cereus, a singleton with new fold. Proteins 2006, 64(I):280-283.

12. Fischer D: Servers for protein structure prediction. Current opinion in structural biology 2006, I 6(2): I 78-182.

13. Siew N, Saini HK, Fischer D: A putative novel alpha/beta hydrolase ORFan family in Bacillus. FEBS Lett 2005, 579(14):3175-3182.
14. Daubin V, Ochman H: Bacterial genomes as new gene homes: the genealogy of ORFans in E. coli. Genome Res 2004, I4(6): 1036-1042.

15. Doolittle RF: A bug with excess gastric avidity. Nature 1997, 388(6642):515-516.

16. Siew N, Fischer D: Unravelling the ORFan puzzle. Comparative and Functional Genomics 2003, 4(4):432-44I.

17. Schmid KJ, Aquadro CF: The evolutionary analysis of "orphans" from the Drosophila genome identifies rapidly diverging and incorrectly annotated genes. Genetics 200I, I 59(2):589-598.

18. Wood V, Rutherford KM, Ivens A, Rajandream MA, Barrell B: A reannotation of the Saccharomyces cerevisiae genome. Comparative and Functional Genomics 200 I, 2(3): I43-I54.

19. Koonin EV, Dolja VV: Evolution of complexity in the viral world: The dawn of a new vision. Virus Res 2006, I I 7(I): I-4.

20. Casjens SR: Comparative genomics and evolution of the tailed-bacteriophages. Curr Opin Microbiol 2005, 8(4):45 I-458.

2I. Juhala RJ, Ford ME, Duda RL, Youlton A, Hatfull GF, Hendrix RW: Genomic sequences of bacteriophages HK97 and HK022: pervasive genetic mosaicism in the lambdoid bacteriophages. J Mol Biol 2000, 299(I):27-5I.

22. Hendrix RW, Smith MC, Burns RN, Ford ME, Hatfull GF: Evolutionary relationships among diverse bacteriophages and prophages: all the world's a phage. Proc Natl Acad Sci U S A 1999, 96(5):2192-2197.

23. Hendrix RW: Bacteriophages: evolution of the majority. Theor Popul Biol 2002, 6 I (4):47I-480.

24. Canchaya C, Fournous G, Brussow $\mathrm{H}$ : The impact of prophages on bacterial chromosomes. Mol Microbiol 2004, 53(I):9-18.

25. Canchaya C, Proux C, Fournous G, Bruttin A, Brussow H: Prophage genomics. Microbiol Mol Biol Rev 2003, 67(2):238-276.

26. Casjens S: Prophages and bacterial genomics: what have we learned so far? Mol Microbiol 2003, 49(2):277-300.

27. Lindell D, Sullivan MB, Johnson ZI, Tolonen AC, Rohwer F, Chisholm SW: Transfer of photosynthesis genes to and from Prochlorococcus viruses. Proc Natl Acad Sci U S A 2004, I01(30): I I013-1 I018.

28. Sullivan MB, Coleman ML, Weigele P, Rohwer F, Chisholm SW: Three Prochlorococcus cyanophage genomes: signature features and ecological interpretations. PLOS Biol 2005, 3(5): I 44.

29. Sullivan MB, Lindell D, Lee JA, Thompson LR, Bielawski JP, Chisholm SW: Prevalence and Evolution of Core Photosystem II Genes in Marine Cyanobacterial Viruses and Their Hosts. PLoS Biol 2006, 4(8):e234.

30. Pruitt KD, Tatusova T, Maglott DR: NCBI Reference Sequence (RefSeq): a curated non-redundant sequence database of genomes, transcripts and proteins. Nucleic acids research 2005, 33(Database issue):D50I-4.

31. Frost LS, Leplae R, Summers AO, Toussaint A: Mobile genetic elements: the agents of open source evolution. Nat Rev Microbiol 2005, 3(9):722-732.

32. Edwards RA, Rohwer F: Viral metagenomics. Nat Rev Microbiol 2005, 3(6):504-510.

33. Skovgaard M, Jensen LJ, Brunak S, Ussery D, Krogh A: On the total number of genes and their length distribution in complete microbial genomes. Trends Genet 200I, I 7(8):425-428.

34. Charlebois RL, Clarke GD, Beiko RG, St Jean A: Characterization of species-specific genes using a flexible, web-based querying system. FEMS Microbiol Lett 2003, 225(2):2 I 3-220.

35. NCBI non-redundant protein DB [ ftp://ftp.ncbi.nih.gov/blast/db/ FASTA/]

36. NCBI environmental non-redundant protein DB [ftp:// ftp.ncbi.nih.gov/blast/db/FASTA/]

37. Yooseph S, Sutton G, Rusch DB, Halpern AL, Williamson SJ, Remington K, Eisen JA, Heidelberg KB, Manning G, Li WZ, Jaroszewski L, Cieplak P, Miller CS, Li HY, Mashiyama ST, Joachimiak MP, van Belle C, Chandonia JM, Soergel DA, Zhai YF, Natarajan K, Lee S, Raphael BJ, Bafna V, Friedman R, Brenner SE, Godzik A, Eisenberg D, Dixon JE, Taylor SS, Strausberg RL, Frazier M, Venter JC: The Sorcerer II Global Ocean Sampling expedition: Expanding the universe of protein families. Plos Biology 2007, 5(3):432-466.

38. Angly FE, Felts B, Breitbart M, Salamon P, Edwards RA, Carlson C, Chan AM, Haynes M, Kelley S, Liu H, Mahaffy JM, Mueller JE, Nulton J, Olson R, Parsons R, Rayhawk S, Suttle CA, Rohwer F: The marine viromes of four oceanic regions. Plos Biology 2006, 4(I I):2|2|-2|3|. 
39. Rohwer F: Global phage diversity. Cell 2003, II 3(2): |4I.

40. Pride DT, Wassenaar TM, Ghose C, Blaser MJ: Evidence of hostvirus co-evolution in tetranucleotide usage patterns of bacteriophages and eukaryotic viruses. BMC Genomics 2006, 7:8.

4I. Kwan T, Liu J, DuBow M, Gros P, Pelletier J: The complete genomes and proteomes of 27 Staphylococcus aureus bacteriophages. Proc Natl Acad Sci U S A 2005, I 02(14):5 I74-5 I79.

42. Kwan T, Liu J, Dubow M, Gros P, Pelletier J: Comparative genomic analysis of 18 Pseudomonas aeruginosa bacteriophages. J Bacteriol 2006, I 88(3): | |84-I | 87.

43. Rokyta DR, Burch CL, Caudle SB, Wichman HA: Horizontal gene transfer and the evolution of microvirid coliphage genomes. J Bacteriol 2006, 188(3): I I34-I I 42.

44. Hatfull GF, Pedulla ML, Jacobs-Sera D, Cichon PM, Foley A, Ford ME, Gonda RM, Houtz JM, Hryckowian AJ, Kelchner VA, Namburi S, Pajcini KV, Popovich MG, Schleicher DT, Simanek BZ, Smith AL, Zdanowicz GM, Kumar V, Peebles CL, Jacobs WR Jr., Lawrence JG, Hendrix RW: Exploring the mycobacteriophage metaproteome: phage genomics as an educational platform. PLoS Genet 2006, 2(6):e92.

45. Moreira D: Multiple independent horizontal transfers of informational genes from bacteria to plasmids and phages: implications for the origin of bacterial replication machinery. Mol Microbiol 2000, 35(I): I-5.

46. Refseq Viral Database [ftp://ftp.ncbi.nih.gov/refseq/release/viral/]

47. NCBI Fully Sequenced Microbial Genomes [ftp:// ftp.ncbi.nih.gov/genomes/Bacteria/]

48. Bose M, Barber RD: Prophage Finder: a prophage loci prediction tool for prokaryotic genome sequences. In Silico Biol 2006 6(3):223-227.

49. Control experiments [http://fischerlab.cse.buffalo.edu/ yin/ phages/viralORFans.html]

Publish with Biomed Central and every scientist can read your work free of charge

"BioMed Central will be the most significant development for disseminating the results of biomedical research in our lifetime. "

Sir Paul Nurse, Cancer Research UK

Your research papers will be:

- available free of charge to the entire biomedical community

- peer reviewed and published immediately upon acceptance

- cited in PubMed and archived on PubMed Central

- yours - you keep the copyright 